\%u Stande komme'), mag in der Melı\%ahl der lialle richtig sein, indessen zoigt unsere Beobachtung, dass die gonorrhoische Infection auch den umgekehrten Weg cinzuschlagen vermag, indem es zuerst zu einer gonorhoischen Vaginitis •kommt.

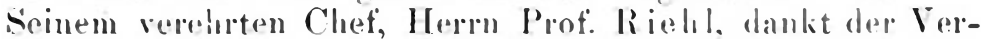
fasser für die in reichem Masse erütiest grewährte Unterstït\%ung sowie für die Ueberlassung dieses seltenen Falles.

VII.

Aus der Privatframenklinik von Prof. Sippel.

\title{
Zwei Fälle von Amputatio Uteri gravidi myomatosi supravaginalis
}

mitgeteilt ron

\author{
I). ERNST PE'TERSEN, \\ Frauenar\%, Frankfurt a. I.
}

Der Lmstand, dass in lem Veit'schen „Handbuch der Grnakologie" aus den Jahren 1885-1896 unter Anführung jedes ein\%elnen, nur t5 Fäle von supravaginaler Amputation des schwangeren, myomatösen Uterus, daron 8 mit tödtlichem Ausgang genannt sind, reranlasst mich \%ur Mitheilung von \%wei derartigen ron Prof. Sippel operirten, gïnstig verlaufenen Fällen, von denen der erstere dem Journal unter mündlicher Frgäinung des Operateurs entnomnten ist, während Operation und Nachbehandlung des zweiten aus eigener Anschaung geschildert sind.

Fall 1. Frau M., 4:3 Jahre alt, kam am 206. August 18:13 \%ur Jehandlung. Die firan hat einmal ror $2(1)$ Jahren \%willinge geloren. seitdem Nenses regelmassig. vierwebentich, nicht sehr stark. let\%te Menses im Mai,

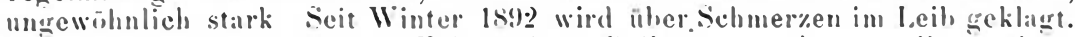
Pat. ist in let\%ter \%eit in Folere einer Reihe von schweren ilensartienen Anfillen schr heruntergekommen und hochgralig abgemagert. Sie muss in die Klinik gretragen werden. Vüllige Appetitlosigkeit und fortwährende? Noigung \%un Erbrechen.

Ber hereits hei der ersten Contersuchung notirte Verdacht aul

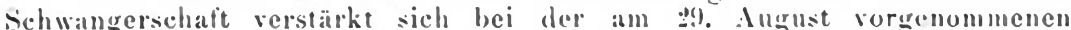
Untersuchung in Narkose Es wird ein kleinkindstioplgrosses. hreithasig vom Fundus des stark vererrisserten, wahrscheinlich graviden Lterus entspringendes Ilrom dignosticirt. Das Ilrom lasst sich frei in der Ballehhöhle bewegen. der Leterus folgr den Bewegungen des Tumors. Die Schmor\%en und ilensartigen Infälle sind entsohieden auf die cxessive Beweglichkeit des Myoms zurück\%üühen. Binde War erfolgrlos angewendet worden.

- Am 1. September 18:13 I, aparitomie in Xarkose (Chloroform. später Billroth'sche Mischung). Es \%eigt sich, dass der lumor, ein etwa zweifaustgrosses Jyom mit hreitem, grosse (iefäsic enthaltendem stiel rom Fundis des schwangeren L̈terus ansegt. Der Leterus selbst ist mit viel-

1) Bum m: Arehir f. (iynaikologie. Nilll, p. 341 . 
fachen kleinen Mromknollen durehsetyt. Supravaginale Amputatio uteri, mit extraperitonealer Versorgung des Stumples.

Glatte Heilung, die freilich dureh eine Thrombose der linken Cruralvene und eine durch die ungünstige Lagerung anf cinem alten scharthantigen 0perationstisch verursalhte trammatische, linksseitige Radialishähmung in die Lünge ge\%ogen wurde. Pat. verlïsst atm 2.2. Oktober dals Bett und wird atm 2. November nach Hause entassen. Die Wunde ist rollstindig geschlossen: Radialislihmung dureh gallatnisehe und faradisehe liehandlung fast gehoben, dis linke Bein schwillt Abends noch etwis an: ron Seiten des Linterleibs keine Beschwerden mehr.

Bei der Wiedervorstellung an 25. Mir\% 18!4 am Banche eine kur\%e Narhe. die Portio als gan\% kleines kinüpfehen \%u liülen.

spiter hildete sich allmählich eine kleine Banchhernie im unteren Theil

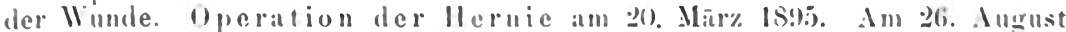
1s:mi wurde dis gunte Resultat der Bruchoperation als andanernd festerestellt. P'at. ist hei rollstindigem Wohlbefinden

In diesem Fall war die Indikation \%ur Entfernung des Nroms per laparatomiam eine vitale, da srehäufte ileusartige Anfäle durch den in der Abdominalhïhle herumfallenden 'Jumor herbeigeführt wurden. Die I)iagnose auf Schwangerschaft wall mit hoher Wahrscheinlichkeit restellt. Eine Alotragunar des arestielten Iroms erschien wegen Grïsse der im Sitiel enthaltenen Crefässe gefährlich, abgesehen davon, dass es bei dem elenden Zustand der Frau nicht zulässig erschien, sie den Gefahren eines erentuell nachfolgenden Aborts aus\%usetren. Die Durchsetzung des lerus mit Mromkeimen war in letater Linie für die liadikaloperation entscheidend. Die Amputatio supraraginalis mit extraperitonealer Stielbehandlung wurde bei schwangerem Cterus, \%umal bei der im Jahre 18933 noch nicht sehr entwickelten 'echnik der Totalexstirpation als die ungefährlichere gewählt.

Fill II. Frau B., 29 Jahre, zuerst vorgestellt den 2.5. Juli 189s. Patt. ist seit Anfang Mai verheiratet. Menses früher regelmässig, nicht anfallend stark. \%uletzt dagewesen den 9. April 1898, cessirten also gleich nach der Verleciratung. Jlie Brüste sollen seit Aushleijen der Menses stäker geworden sem, sonst keinerlei subjective Schwingerschafts\%cichen seit 14 Tagen wird über starke krampfartig auftretende, in das rechte lBein ausstrahlende schmer\%en in der rechten Seite des Cuterleibs geklant: ïlnliche Schmer\%en geringeren Grades sollen früher leei der Periode anfigetreten sein.

Befund: Im Unterleib in der Nitte liegend ein links einen Querlinger über den Niblel, rechts etwis weniger hoch reichender, ans drei dhteilungen ron versehiedener Consisten\% bestehender, nirgends druckemplindlicher Tumor. Die eine grössere Ahteilung links oben nach vorn \%u liegend hat die Consisten\% einer Cyste, die andere Abteilung rechts, mehr \%urickliegend und kleiner als die erste, hat ebenso wie die zwischen und unter heiden liegende dritte eine feste Consisten\%. Die Tumoren sind nicht gogen einander abzugren\%en. nur durch furchen getremnt.

In der minsig weiten Scheide wird dats vordere Scheidengewïlbe und die rordere Scheidenwand durch einen harten. ctwas mehr rechts liegenden Tumor ron der Grösse eines kleinen lindskopfes his dieht hinter den Introitus herabgedrängt. Der Tumor erweist sich als fortset\%ung der miteleren der drei von Aussen gefuhlten Abteilungen. Die Portio mit dem gräbehenformigen Muttermund ist nach hinten uad ohen gredrängt. Die libase liegt rechts unter dem Tumor. Die Cervix litsst sich bimanuell ungefähr $2 \mathrm{~cm}$ weit nach rechts hinten olsen verfolgen. Vagina feicht venos verfirlut. Dic Brüste enthalten Colostrum.

Diagnose: Schwangarschatit des mromatisen Cterus. wobei die links rorn liegende Abteilung als schwangerer Literus angesprochen wird. 
Pat. Wird \%unächst nach Hause entlassen, mit der Weisung, sich in 14 lagen wieder vor\%stellen; es soll versucht werden. das Finde der Schwangerschalt ab\%uwarten. Statt dessen muss sie bereits am 4. August in die lilinik aufgenommen werden, weil ihr \%ustand sich ansserordentich rerschlimmert hat. Die. Tumoren verursachen schr heftige Schmermen; Pationtin macht einen sehr elenden kindruck.

Den 6. August 189S Operation: Aethernarkose. Bauchschnitt. Der 'Lumor verhailt sich genau wie diagnosticirt. Ausserdem findet sich noch ein drittes grosies ron der hinteren Literuswand ausgehendes Myom. Der schwangere Lterus selbst ist ron \%ahlreichen Jyomkeimen resip. -knollen durehset\%. Dic ligamental lata werden beiderseits nach doppeler Lnterbindung durchtrennt, dann das praecervikale Myom nach querer spaltung des Peritonealiabermuges aus dem Beckenbindegewebe enucleirt. Nachdem (1) cine stielbildung crmöglicht war, wird nach Inlegen der elastischen ligatur die: suplaviginale I mputation ausgefinhr. Jistraperitoneale sticllukandlnng wie bei liall 1 .

Villigr reactionsloser Verlauf. Die Temperatur erreicht eimmal am S. Tage $37, \%$ soust stets \% wischen $36,4-37,4$. Der \%iemlich dicke Stumpt liist sich langsam, so dass er erst am 3. Septlur. (t Wochen post operationem? abgehohen werden kann. Der in der Tiefe versehwindende Stiol hinterlisst einen \%iemlich tiefen Trichter. Erstes Infstehen den 10 Sepremlier. l3ei der an 17. sieptember erfolgten lintlassung ist an Stelle des Stiels eine grut. uramulirende, in der Tiefe geschlossene, wenige cm grosse Batuchuarle vorhanden. Pat. hei vor\%üglichem Wohlhetinden, ohne Beschwerden und hei bedeutend hesserem Ausselien und broähungs\%usand als vor der Operation.

Auch in diesem Fall war der Grund zum Eingriff eine Indicatio vitalis, diesmal jedoch ausgelöst durch linklemmungserscheinungen des 'lumors im kleinen Becken. Vor dem Manifestwerden dieser Frscheinungen war, wie schon gesant, bei der. sicher diagnosticirten Schwangerschaft ruhiges dhwarten beabsichtigt, um am Ende derselben eventuell durch Kaiserschnitt ein lebendes lind \%u erhalten, woran sich wahrscheinlich abdominelle Totalexstirpation hätte anschliessen müssen. Es wurde die supravaginale Imputation mit extraperitonealer Stiellbelandlung gewählt, weil einerseits der Uterus ausser den diagnosticirten Mromen sehr \%ahlreiche mehr oder minder grosse Myomkeime resp. -knollen aluies und deshall, ron Enucleation keine Rede seine konnte und weil andererseits die schlecht vertragene Narkose eine rasche Beendigung der Operation nöthig machte.

Wire es nicht so selur auf Abkürmug der Operations\%eit angekommen, so hätten wir die Totalexstirpation, die wir unter den Radikaloperationen bei Myom principiell als die rollkommenste Operationsweise berorzugen, ausgeführt

Die Eigenart unseres Materials hat uns jedoch schon vielfach in die lange gebracht, auf die Totalexstirpation vermichten \%u müssen. Gründe lokaler Art lassen die Frauen mit Myomen fust stets erst in sehr vorgeschritteuen Stadium, mit geschwäichter Her\%kraft in unsere klivische Behandlung treten. Dass aber unter solchen Linstainden grade durch die supraraginale Amputation mit extraperitonealer Stielbehaudlung gute Resultate er\%ielt werden kïnnen, beweist eine Serie ron mehr als ?() in der Sippel'scheu Kilinik nach dieser. Methode behandelten Fällen, die nicht nur alle mit Heilung endigten, sonderu auch ohne jede Stürung verlieten. 
Der entfernte Cterus des 2. Falles ist ein selten schünes Priparat. Er erregte durch soin eigenartigres Verhalten nach der Ahtragung unser besonderes Interesse. Während nimlich in Augenblick der Aloset\%ung: dic noch in der Cervix erfolgte, rom lii nichts sichthar watr, da ungefïhr $1 \mathrm{~cm}$ Cervikalkanal erhalten war, iffnete sich der Cervixrest noch in der Hand des Operateurs. Es entstand unter sichtbarer Retration der Cervicialwand eine (al. 10pfennigstückgrosse Oeflumng, atus der sich die bihäute hervordränten. Diese brweiterung verstäkte sich an dem anstreschnittenen Organ Wiltrend der \%eit, die auf Versorgung des Stumpfes und Schluss der Bauchwunde verwendet wurde, so sehr, dass hei Besichtigung des Priparates nach rollendeter Operation die Cervicalïfinung reichlich ómarkstückgross nar, wahrend die Eihäute sich wie ein Grinseei, das \%ur halben Höhe im bibecher steckt, als Fruchthlase ans ihm herrorwöben.

Das Priparat wurde atm 1!). September 1598 ron Prof. Sippel im Arrotlichen Verein Franklurt at. II rorge\%eigt.

lis hat jet folgendes Aussehen: Beimahe mamnskopigrosser Tumor, ron rorn gestlien alus 2 Hauptahteilungen bosteliend, einer linken gröisseren. durch den Abgang der Tuben als eigenticher Lterus gekenn\%eichnet, ron 16 mehr minder grossen Myomknoitchen und -knollen durehset\%t, und einer kleineren rechten, die durch ein massires Myom gebildet ist Die Suhstan\% des eigentichen Corpus set\%t sich nach unten rorn in ein sich schrigg ron links nach rechts erstreckendes, also praccervical gelegenes, dem ersterenannten an Griosse etwa gleiches Jrom fort. Tieses ist in seinem interen Theil ron Peritoneum entblïsst und durch die Schnittliache unten begrenz. An der hinteren Flatehe springt, etwi ron der Mitte des Fundus ansrehend, win weiteres fotst kleinkindskopfurosses MYom vor. Die untere Fliche bildet die schnittlähe des pratecervicalen llyoms: hinter dieser, etwas nach rechts verschoben und in einer etwals höheren Ehene der rundlich ovale, nach Geffinung der lihäute an dem in formol gehiarteten l'riipatrat, noch reichlich thalergrosse innere Muttermunc. Durch die Definung der Eihailute sieht man in die sich schriig nach links in die Hohe erstreckende Eternshühle und erthlickt in dieser den mit den fü̈sen nach unten liegenden Foetus.

Ilerr Prof. Sippel knüpte an die Vor\%ecigung des Präparats die Bemerkung, dass die eigenthümlich expulsive Wirkung des abgeschnittenen Literus anf seinen Inlialt vielleicht greeignet sei, cin neues hicht auf den bisher noch riemlich unaufgeklärten Vorgang der nachtrïglichen Ausstossung von Früchten unentlunden verstorbener Mlitter \%u werfen.

Han nalin bekanntlich bisher' an, dass dieses l'actum sich ahspiele durch den Iruck der liaulnissgase, dic sich in der Abdominalhohle entwickelten. Der ron uns beobachtete Vorgang dürfte vermuten lassen, dass hierbei anch noch andere kirafte wirksam sind, dass auch liei den Leichengeburten der Ëterus sellsit eine gewisse expulsise Kraft entfaltet, die wahrscheinlich darauf \%urückuführen ist, dass die bei quergestreiften Muskeln meist erst spaiter als "Leichenstarre" anftretende Verküroung in den glatten Muskelfasern des Lterus früheitiger in Erscheinung tritt. Es wïrde dies ein Anilogon sein \% dem häufig beobachteten Abgang ron lioth, Urin etc. kur\% vor oder im Voment des Todes, Vorgainge die auch wohl kilum durch die Erschlatfiung der entsprechenden Sphincteren allein erklärt werden können, souder'n ebenfalls auf tonische Contraction glatter Muskelfasern \%urückgefïhrt werden muissen. ${ }^{2}$ )

1) Schroeder, Lehrbuch d. Geburtshäle. T. Anflage. pate. ti3.

2) Nach Niederschrift dieses tinde ich im Centrallhatt für Grinatecologie No. $2 S$ ein Referat über eine Veroffentlichug ron Bleich (Tschirniul). - Ceber Sarggeburt und Nittheilung eines neuen Falles“ aus der Vierteljahrsschrift für gerichtliche Medicin. 18!\%. Heft t, in weleher der Verfisser sich in ähnifiem Sinne ausspricht, indem er \%um Zustandekommen einer Sargoburt neben pracmortalen Uteruscontractionen - Wehen - auch posimortale lirifte - Fäulnissgase und tonische Cieruscontractionen - ils nothwendig annimmt. 